\title{
Back Radiation Suppression through a Semitransparent Ground Plane for a mm-Wave Patch Antenna
}

\author{
Kirill Klionovski and Atif Shamim, Senior Member, IEEE
}

\begin{abstract}
Omnidirectional radiation pattern with minimum backward radiation is highly desirable for base station antennas to minimize the multipath effects. Semitransparent ground planes have been used to reduce the backward radiation, but mostly with complicated non-uniform impedance distribution. In this work, we propose, for the first time, a round semitransparent ground plane of radius $0.8 \lambda$ with uniform impedance distribution that can improve the front-to-back ratio of a wideband patch antenna by 11.6 dB as compared to a similar sized metallic ground plane. The value of uniform impedance is obtained through analytical optimization by using asymptotic expressions in the Kirchhoff approximation of the radiation pattern of a toroidal wave scattered by a round semitransparent ground plane. The semitransparent ground plane has been realized using a low-cost carbon paste on a Kapton film. Experimental results match closely with those of simulations and validate the overall concept.
\end{abstract}

Index Terms-ground plane, impedance, optimization, wideband patch antenna.

\section{INTRODUCTION}

$\mathrm{N}$ EW millimeter-wave and $5 \mathrm{G}$ telecommunication technologies are required to handle large mobile data traffic through a large number of antennas [1-3]. Due to the large number of antennas that will be operating simultaneously in a real environment, the quality of radio signals is expected to suffer considerably due to multipath effects [4]. The multipath effect, where a radio signal reaches the antenna by two or more paths due to its reflection from different objects or the earth's surface near the antenna, can reduce the signal to noise ratio and increase the bit error rate for digital signals. Thus, for base station antennas, important considerations are wide bandwidth to maintain high data rates and controlled specific shaped radiation patterns to reject or reduce the multipath effects. Ideally, these antennas must demonstrate an omnidirectional radiation pattern in the upper half-sphere and almost zero radiation in the backward direction, which means they must attain the best possible front-to-back ratio (FBR). Therefore, a patch antenna could be a very suitable candidate for base station antennas.

Typically, large metallic ground planes are used to improve the FBR of a patch antenna. It was shown in [5] that the FBR of a patch antenna with a round metallic ground plane does not improve beyond a certain size of ground plane. Some researchers have tried to use other types of ground planes [6], such as choke rings and impedance ground planes, but these structures are quite narrow-band. The concept of round, semitransparent ground planes was introduced in [7-11] to provide wide-band suppression of the backward radiation of patch antennas. In [7], a Global Positioning System (GPS) base station patch antenna design is shown, which benefits from a semitransparent ground plane realized through complicated Rcard technology. It utilizes a ground plane with a radius of 6.5 inches with a non-uniform isotropic resistive impedance distribution. In references [8, 11], a semitransparent ground plane with a radius of 13 inches with a non-uniform isotropic resistive impedance distribution has been realized. The design targets the GPS band and utilizes an artificial magnetic conductor approach. In [9], a GPS patch antenna is shown, which utilizes a semitransparent ground plane with a radius of $150 \mathrm{~mm}$ and a non-uniform anisotropic inductive impedance. This surface has been realized by using a periodic structure of metal strips, with chip elements in the gap between the strips. In [10], a GPS patch antenna with a semitransparent ground plane with a radius of $80 \mathrm{~mm}$ and non-uniform isotropic resistive impedance distribution has been demonstrated. The resistive layer has been realized using LLumar film.

In all the above examples [7-11], the proposed ground planes with non-uniform impedance distributions have been realized for GPS frequency band 1-1.7 GHz. Fabrication of such types of non-uniform semitransparent surfaces, with a wide range of values of impedances along the radius of a ground plane, is quite difficult in the millimeter-wave band. On the other hand, the uniform impedance distribution is relatively easy to realize, however it has never been reported in the literature.

In the present paper, we investigate, for the first time, a wideband patch antenna that has a round, semitransparent ground plane of radius $0.8 \lambda$ ( $\lambda$ is wavelength) with a uniform isotropic resistive impedance distribution. We theoretically optimize the value of the impedance to maximize the FBR of the design. For this purpose, we have used asymptotic formulae for the radiation pattern of a toroidal wave scattered by a round, 
semitransparent ground plane. Also, we present experimental radiation patterns and $S_{11}$ of the patch antenna with semitransparent and metallic ground planes. The comparison of the proposed design with a similar patch antenna (but metallic ground plane) confirms the better suppression of back radiation by the former.

\section{The Optimization Of TRANSPARENCY Of THE GRound Plane With The Patch ANTENNA}

Let us consider a patch antenna with radius $a$, which is excited by a coaxial cable and located above a metallic ground plane with radius $R$ at distance $h$ (Fig. 1). We assume that the radiation pattern of the patch antenna without the ground plane

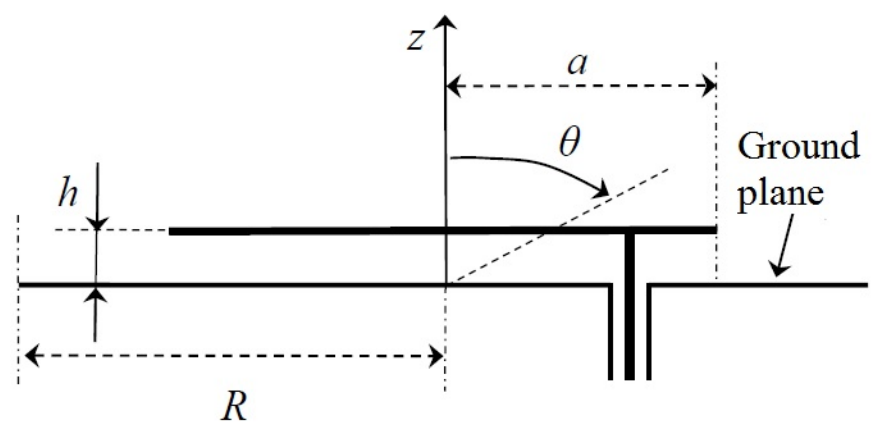

Fig. 1. The patch antenna with the round ground plane. Side view.

coincides with the radiation pattern of an annular magnetic current with a single azimuthal variation of amplitude $[9,12]$ in cylindrical coordinates $(\rho, \varphi, z)$ :

$$
\mathbf{j}_{m}(\rho, \varphi, z)=I_{m} \delta(\rho-a) \delta(z-h) \cos (\varphi) \boldsymbol{\varphi}_{0} .
$$

Here, $\delta(z)$ is the Dirac delta-function of argument $z ; \varphi_{0}$ is the unit vector in the $\varphi$-direction; and $I_{m}$ is the amplitude of the magnetic current. Far field of the annular current (1) in free space has the components of the magnetic field intensity vector in spherical coordinates $(r, \theta, \varphi)$ [5]:

$$
\begin{aligned}
& H_{\theta}(r, \theta, \varphi)=\frac{I_{m} k a}{i 4 W_{0}} \cos \theta \sin \varphi \times \\
& \times\left(J_{0}(k a \sin \theta)+J_{2}(k a \sin \theta)\right) \frac{e^{-i k r}}{r}, \\
& H_{\varphi}(r, \theta, \varphi)=\frac{I_{m} k a}{i 4 W_{0}} \cos \varphi \times \\
& \times\left(J_{0}(k a \sin \theta)-J_{2}(k a \sin \theta)\right) \frac{e^{-i k r}}{r},
\end{aligned}
$$

where $k=2 \pi / \lambda, i=\sqrt{-1}, W_{0}=120 \pi$, and $J_{n}(x)$ is the Bessel function of order $n$ and argument $x$. The backward radiation of the annular magnetic current with a round metallic ground plane for angles $\theta$ near the $z$-axis in asymptotic Kirchhoff approximation is created by edge current of the ground plane $[5,6]:$

$$
\mathbf{j}_{e}(\rho, \varphi, z)=\left(j_{\rho}(R, \varphi) \boldsymbol{\rho}_{0}+j_{\varphi}(R, \varphi) \boldsymbol{\varphi}_{0}\right) \delta(\rho-R) \delta(z),
$$

where $\boldsymbol{\rho}_{0}$ is the unit vector in the $\rho$-direction, $j_{\rho}(R, \varphi)$ and $j_{\varphi}(R, \varphi)$ are functions representing the radial and azimuthal electric currents on the edge of the ground plane. These functions have the form:

$$
\begin{aligned}
& j_{\varphi}(\rho, \varphi)=\frac{i I_{m} k a}{2 W_{0}} \sin \left(\theta^{s}(\rho)\right) \frac{e^{-i k \sqrt{\rho^{2}+h^{2}}}}{\sqrt{\rho^{2}+h^{2}}} \sin \varphi \times \\
& \times\left(J_{0}\left(k a \cos \left(\theta^{s}(\rho)\right)\right)+J_{2}\left(k a \cos \left(\theta^{s}(\rho)\right)\right)\right), \\
& j_{\rho}(\rho, \varphi)=\frac{i I_{m} k a}{2 W_{0}} \frac{e^{-i k \sqrt{\rho^{2}+h^{2}}}}{\sqrt{\rho^{2}+h^{2}}} \cos \varphi \times \\
& \times\left(J_{0}\left(k a \cos \left(\theta^{s}(\rho)\right)\right)-J_{2}\left(k a \cos \left(\theta^{s}(\rho)\right)\right)\right), \\
& \theta^{s}(\rho)=\arctan \left(\frac{h}{\rho}\right) .
\end{aligned}
$$

The radiation pattern of the annular electric current (3) has the components [5]:

$$
\begin{aligned}
& H_{\theta}^{e}(\theta, \varphi)=\left[j_{\varphi}(R, \varphi)\left(J_{0}(k R \sin \theta)-J_{2}(k R \sin \theta)\right)-\right. \\
& \left.-j_{\rho}(R, \varphi)\left(J_{0}(k R \sin \theta)+J_{2}(k R \sin \theta)\right)\right] \frac{i k R}{4}, \\
& H_{\varphi}^{e}(\theta, \varphi)=\left[j_{\varphi}(R, \varphi)\left(J_{0}(k R \sin \theta)+J_{2}(k R \sin \theta)\right)-\right. \\
& \left.-j_{\rho}(R, \varphi)\left(J_{0}(k R \sin \theta)-J_{2}(k R \sin \theta)\right)\right] \cos \theta \frac{i k R}{4} .
\end{aligned}
$$

The components of the radiation pattern of the annular magnetic current above the metallic ground plane of radius $R$ for angle $\theta=\pi$ are derived by using (4) and (5):

$$
\begin{aligned}
& H_{\theta}^{e}(\theta=\pi)=\frac{I_{m} k^{2} a}{16 W_{0}} \frac{e^{-i k \sqrt{R^{2}+h^{2}}} R}{\sqrt{R^{2}+h^{2}}} I_{\varphi \rho}, \\
& H_{\varphi}^{e}(\theta=\pi)=-\frac{I_{m} k^{2} a}{16 W_{0}} \frac{e^{-i k \sqrt{R^{2}+h^{2}}} R}{\sqrt{R^{2}+h^{2}}} I_{\varphi \rho}, \\
& I_{\varphi \rho}=-J_{0}\left(k a \cos \left(\theta^{s}(R)\right)\right)+J_{2}\left(k a \cos \left(\theta^{s}(R)\right)\right)+ \\
& +\sin \left(\theta^{s}(R)\right)\left(J_{0}\left(k a \cos \left(\theta^{s}(R)\right)\right)+J_{2}\left(k a \cos \left(\theta^{s}(R)\right)\right)\right) .
\end{aligned}
$$

We can simplify (6) when $R \rightarrow \infty$ :

$$
\begin{aligned}
& H_{\theta}^{e}(\theta=\pi)=-\frac{I_{m} k^{2} a}{16 W_{0}}\left(J_{0}(k a)-J_{2}(k a)\right) e^{-i k \sqrt{R^{2}+h^{2}}}, \\
& H_{\varphi}^{e}(\theta=\pi)=\frac{I_{m} k^{2} a}{16 W_{0}}\left(J_{0}(k a)-J_{2}(k a)\right) e^{-i k \sqrt{R^{2}+h^{2}}}
\end{aligned}
$$

We can see from (7) that amplitude of the backward radiation of the annular magnetic current above the metallic ground plane depends on the radius of the magnetic current and does not 
depend on the radius of the ground plane. Physically, it can be explained by the fact that amplitude of the radial electric current $j_{\rho}(R, \varphi)$ on the edge of the metallic ground plane, which is created by the field (2) of the magnetic current (1), decreases proportionally $R$ after a certain size. At the same time, the amplitude of the components of the radiation pattern (5) of the electric edge current (3) increases proportionally $R$. As a result, the amplitude of the backward radiation is constant when $R \rightarrow \infty$.

To reduce the backward radiation, we will use a round, semitransparent ground plane. A semitransparent surface is generally characterized by the fact that an incident plane electromagnetic wave partially reflects and partially passes through the surface. For a two-dimensional round ground plane located in polar coordinates $(\rho, \varphi)$, this surface is characterized by two reflection coefficients, $\eta_{\rho, \varphi}$, and two transmission coefficients, $\tau_{\rho, \varphi}=1-\eta_{\rho, \varphi}$ (for waves with radial $(\rho)$ and azimuthal $(\varphi)$ components of the magnetic field intensity vector on the ground plane's surface). In the axisymmetric case, these coefficients depend only on radial coordinate $\rho$ on the ground plane's surface and the angle of incidence of an incident wave onto the ground plane at this point. Impedance of the semitransparent surface, $Z_{\rho, \varphi}$, relates to the reflection coefficient as $\eta_{\rho, \varphi}=60 \pi /\left(Z_{\rho, \varphi}+60 \pi\right)$. The task of scattering a toroidal wave by a round, semitransparent ground plane was investigated in [6]. In that paper, it was shown that if the reflection and transmission coefficients are slowly varying functions along the radial coordinate $\rho$, the backward radiation near the $z$-axis of a ground plane is created by the edge current of the ground plane.

Let us consider a model of the annular magnetic current (1) located above a semitransparent ground plane of radius $R$ at distance $h$. We assume that the ground plane has a central metallic area with a radius of $R_{1}$, which is more than the radius of the annular magnetic current, $Z_{\rho, \varphi}(\rho)=0$ for $0 \leq \rho<R_{1}, a<R_{1}$, and a periphery area with uniform isotropic resistive impedance, $Z_{\rho}(\rho)=Z_{\varphi}(\rho)=A$ for $R_{1} \leq \rho \leq R$. For this model, components of the radiation pattern, for angles $\theta$ near the $z$-axis in asymptotic Kirchhoff approximation, are created by two annular electric currents of form (3) and radii of $R_{1}$ and $R$ :

$$
\begin{aligned}
& H_{\theta}^{s}(\theta, \varphi)=\left[I_{\varphi}\left(R_{1}, \varphi\right)\left(J_{0}\left(k R_{1} \sin \theta\right)-J_{2}\left(k R_{1} \sin \theta\right)\right)-\right. \\
& \left.-I_{\rho}\left(R_{1}, \varphi\right)\left(J_{0}\left(k R_{1} \sin \theta\right)+J_{2}\left(k R_{1} \sin \theta\right)\right)\right] \frac{i k R_{1}}{4}+ \\
& +\left[I_{\varphi}(R, \varphi)\left(J_{0}(k R \sin \theta)-J_{2}(k R \sin \theta)\right)-\right. \\
& \left.-I_{\rho}(R, \varphi)\left(J_{0}(k R \sin \theta)+J_{2}(k R \sin \theta)\right)\right] \frac{i k R}{4}, \\
& H_{\varphi}^{s}(\theta, \varphi)=\left[I_{\varphi}\left(R_{1}, \varphi\right)\left(J_{0}\left(k R_{1} \sin \theta\right)+J_{2}\left(k R_{1} \sin \theta\right)\right)-\right. \\
& \left.-I_{\rho}\left(R_{1}, \varphi\right)\left(J_{0}\left(k R_{1} \sin \theta\right)-J_{2}\left(k R_{1} \sin \theta\right)\right)\right] \frac{i k R_{1}}{4} \cos \theta+ \\
& +\left[I_{\varphi}(R, \varphi)\left(J_{0}(k R \sin \theta)+J_{2}(k R \sin \theta)\right)-\right. \\
& \left.-I_{\rho}(R, \varphi)\left(J_{0}(k R \sin \theta)-J_{2}(k R \sin \theta)\right)\right] \frac{i k R}{4} \cos \theta,
\end{aligned}
$$

where amplitudes of radial and azimuthal annular electric currents have the form $I_{\rho, \varphi}(\rho, \varphi)=\eta_{\varphi, \rho}(\rho) j_{\rho, \varphi}(\rho, \varphi)$. Fig. 2 shows, by
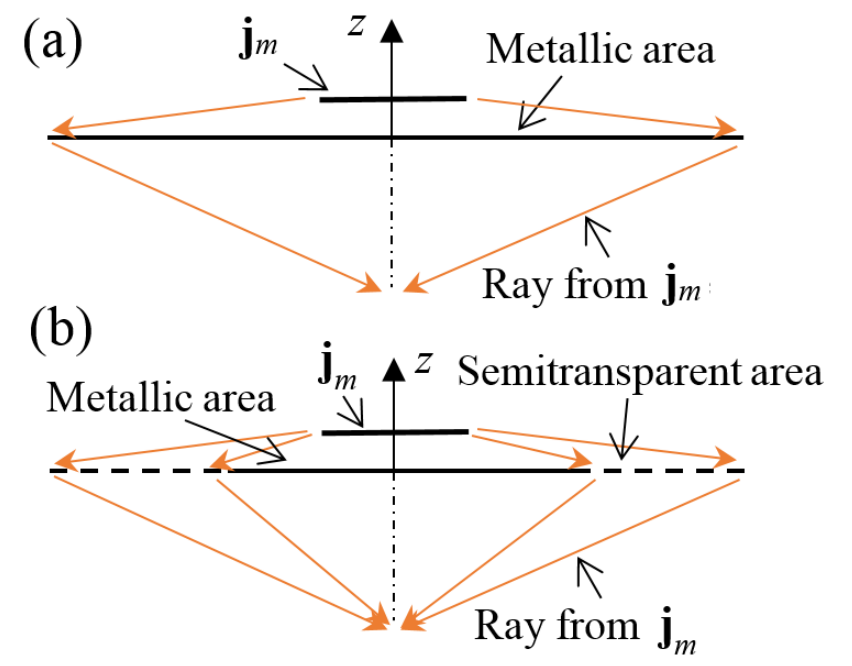

Fig. 2. The propagation of rays from the annular magnetic current above the metallic (a) and semitransparent (b) ground plane.

using a propagation of electromagnetic waves in the form of rays, how backward radiation is created at angle $\theta=\pi$ for the model of the annular magnetic current above the metallic and semitransparent ground plane.

Let us optimize the parameters $A$ and $R_{1}$ of the ground plane with a radius of $0.8 \lambda$ to decrease the backward radiation of the annular magnetic current with the parameters $a=0.14 \lambda, h=0.05 \lambda$. For this case, we consider a goal function that is the mean square power radiated in the sector of angles $160^{\circ} \leq \theta \leq 180^{\circ}$ :

$$
P_{b}=\int_{8 \pi / 9}^{\pi} \int_{0}^{2 \pi}\left(\left|H_{\theta}^{s}(\theta, \varphi)\right|^{2}+\left|H_{\varphi}^{s}(\theta, \varphi)\right|^{2}\right) d \varphi d \theta
$$

After a substitution of formulas (8) into (9) and the minimization of the goal function using the nonlinear conjugate gradient method, we obtain the next optimum values of the amplitude of the impedance and the radius of metallization: $A=72 \mathrm{Ohm}, R_{1}=0.38 \lambda$. Note that minimization of the backward radiation of an annular magnetic current above a semitransparent ground plane of radius $0.8 \lambda$ was provided in [13], and the optimized distribution of a non-uniform anisotropic resistive impedance was presented there.

\section{DESIGN AND RESULTS}

\section{A. The Design and Fabrication of the Patch Antenna with the Ground Planes}

In Section II, a circular patch antenna with a semitransparent ground plane has been used for optimization of the semitransparent ground plane surface impedance. But circular patch antenna is a narrow-band antenna [12], and for typical mm-wave applications, such as 5G or Wireless Personal Area Network (WPAN), larger bandwidths are required. For example, 5G base station antenna is required to have a bandwidth of approximately 10\% [14]. For bandwidth enhancement, we will use a design of a wide-band stacked patch antenna (Fig. 3), which is described in [15]. It is worth mentioning here that the theory developed in Section II works 
(a)

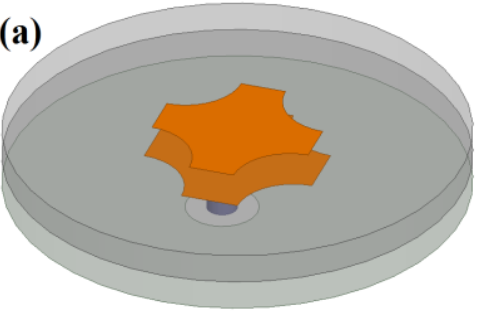

(b)

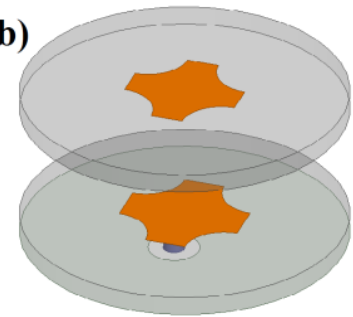

Fig. 3. Stacked patch antenna (a) and two separated layers of the antenna (b).

equally well with this modified shaped patch antenna (as will be shown later in comparison between simulations and measurements). A stacked wide-band patch antenna with round, semitransparent, and metal ground planes (Fig. 4) with a radius of $8 \mathrm{~mm}$ was fabricated and measured to verify the results of the optimization. The patch has two dielectric layers of the (a)

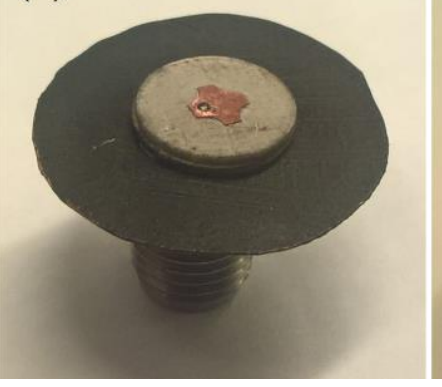

(b)

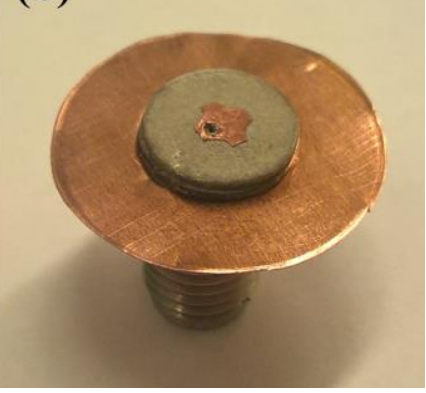

Fig. 4. Photo of the patch antenna with the semitransparent (a) and metallic (b) ground plane.

Rogers RO3003 laminate with a thickness of $0.5 \mathrm{~mm}$ and a radius of $3.8 \mathrm{~mm}$ for each layer. Each layer has a square metal plate with angled circular cutouts. The centers of the cutouts are located at the tops of the squares. The lengths of the bottom and the top squares are 2.85 and $2.65 \mathrm{~mm}$ respectively. The radius of all cutouts is $0.95 \mathrm{~mm}$. The patch is excited by a $50-\Omega$ coaxial cable, which is located $0.78 \mathrm{~mm}$ from the axis of the patch. The central core of the coaxial cable has electric contact with only the bottom plate.

The semitransparent ground plane was fabricated using R-

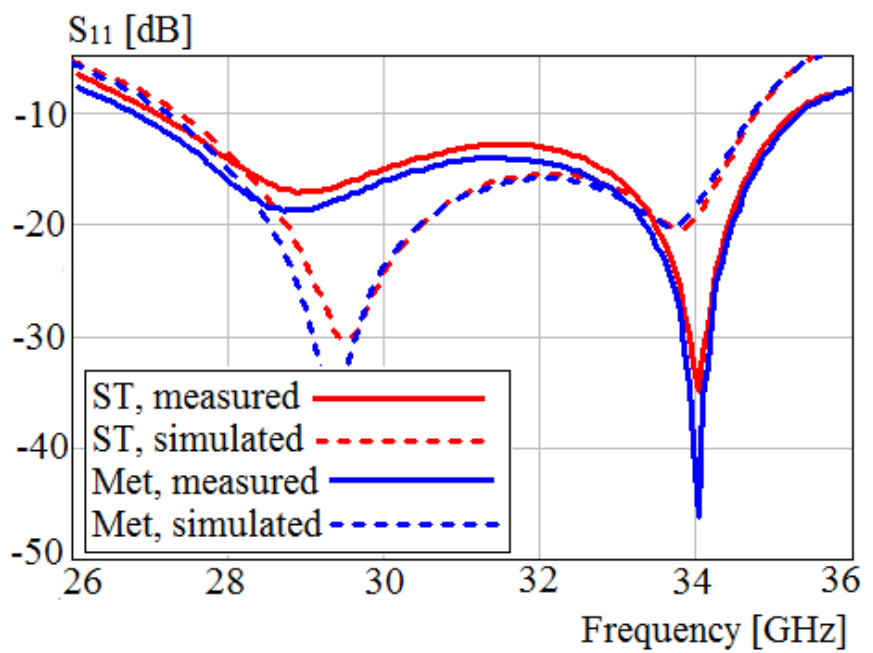

Fig. 5. $\mathrm{S}_{11}$ of the patch antenna with the ground planes.

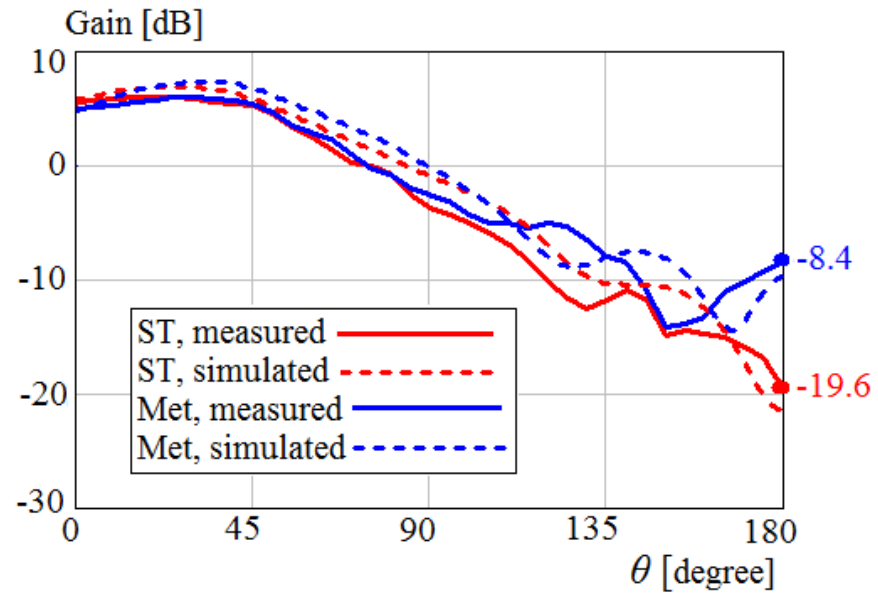

Fig. 6. Radiation patterns of the patch antenna with the ground planes in the E-plane at $30 \mathrm{GHz}$.

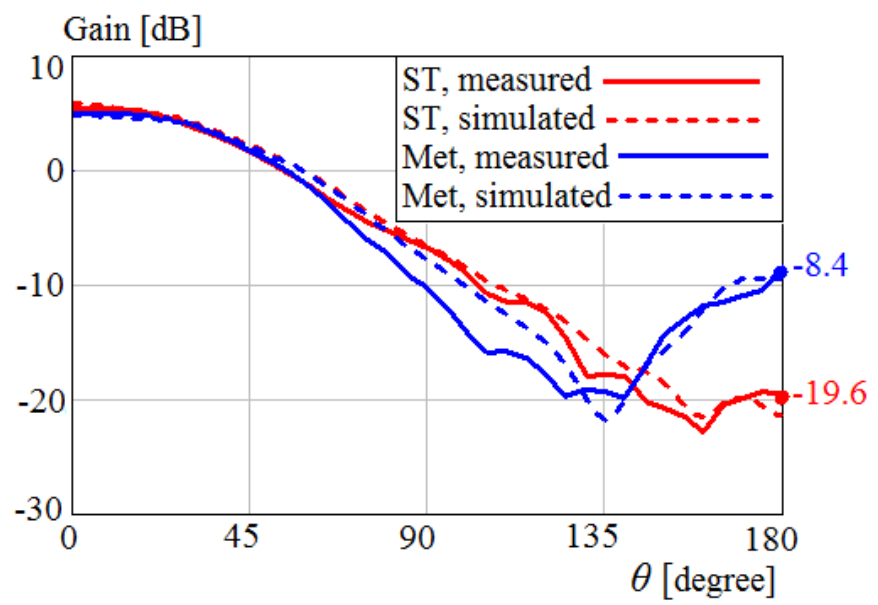

Fig. 7. Radiation patterns of the patch antenna with the ground planes in the $\mathrm{H}$-plane at $30 \mathrm{GHz}$.

card technology by spreading a thin layer of carbon paste with uniform thickness on a thin Kapton film. The value of the impedance and the inner radius of the $\mathrm{R}$-card is $70 \mathrm{Ohm}$ and 3.8 $\mathrm{mm}$ respectively. This value was measured using the waveguide technique [16].

\section{B. Measurements Results}

Figs. 5-9 plot $S_{11}$ and the measured and simulated radiation patterns of the antenna with the semitransparent (ST) and metallic (Met) ground planes at frequencies of 27 and $30 \mathrm{GHz}$.

Simulations were performed using Ansoft HFSS software for the dimensions mentioned above. The ground planes radii are $0.8 \lambda$ and $0.72 \lambda$ for frequencies of 30 and $27 \mathrm{GHz}$ respectively. Although the optimization procedure in Section II has utilized a ground plane of radius $0.8 \lambda$, it has been confirmed that the ground plane of radius $0.72 \lambda$ also works well and the FBR for the two ground plane sizes is not much different (the values are given in the next paragraph).

Fig. 5 shows that $S_{11}$ is less than $-10 \mathrm{~dB}$ for the band 27-35.4 $\mathrm{GHz}$ (the bandwidth is 27\%) and is similar for both types of ground planes. Generally, there is good agreement between simulated and measured results. However, there is some 


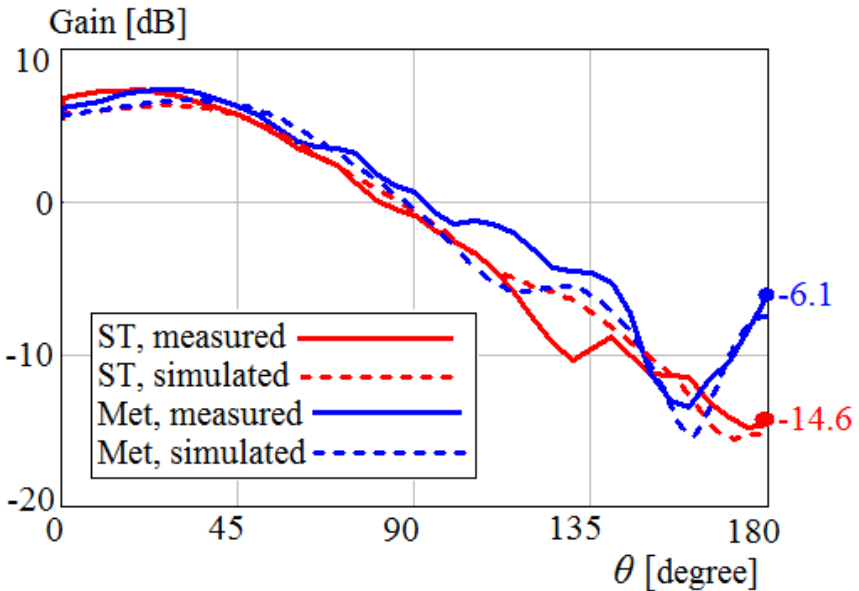

Fig. 8. Radiation patterns of the patch antenna with the ground planes in the E-plane at $27 \mathrm{GHz}$.

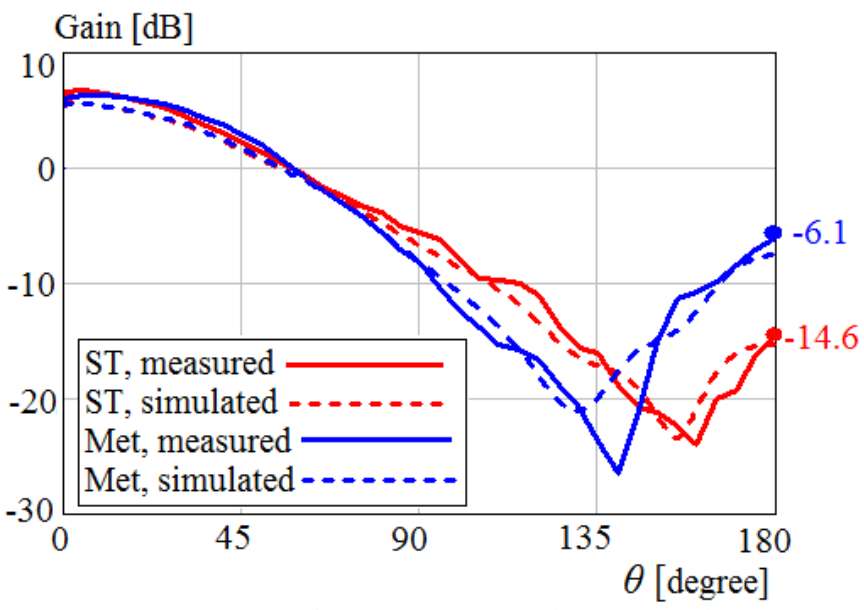

Fig. 9. Radiation patterns of the patch antenna with the ground planes in the H-plane at $27 \mathrm{GHz}$.

discrepancy between the simulated and measured results, particularly at the resonance points $(29.5$ and $34 \mathrm{GHz})$ and this could be due to the tolerance in the material properties of the material (RO3003 laminate) at higher frequencies. The material properties used in the simulator are given for $10 \mathrm{GHz}$ and it is likely that these values are somewhat different at higher frequencies, thus causing this small discrepancy. Part of this variation may be due to the fabrication tolerances. Nonetheless, the antenna is well matched for the entire bandwidth and this variation is not detrimental to antenna performance. Figs. 6-9 show that FBR is 25.1 and $13.5 \mathrm{~dB}$ for the patch antennas with the semitransparent and metallic ground planes of radii $0.8 \lambda$ respectively, and FBR is 21.3 and $12.4 \mathrm{~dB}$ for the patch antenna with the semitransparent and metallic ground plane of radius $0.72 \lambda$ respectively. The backward radiation of the patch antenna with the semitransparent ground plane is less than with the metallic one for nearly all angles $\theta$ in the E-plane. In the $\mathrm{H}$ plane, the backward radiation of the patch antenna with the semitransparent ground plane is less than with the metallic one for the angles $145^{\circ} \leq \theta \leq 180^{\circ}$. The value of the radiation pattern of the patch antenna in the E-plane at the angle $\theta=90^{\circ}$ is $1.5 \mathrm{~dB}$ more with the metallic ground plane than with the semitransparent one. In the H-plane, there is the opposite conclusion.

We can see also that the increase of gain is 0.44 and $0.35 \mathrm{~dB}$ for the patch antenna with the semitransparent ground plane of radius $0.8 \lambda$ and $0.72 \lambda$ respectively, in comparison with the metallic ground plane of the same radius. Thus, reducing the backward radiation of the patch antenna with the semitransparent ground plane, in comparison with the metallic one, results in the gain increasing; but not only the energy losses in the resistive layer of the ground plane.

\section{Dual-polarized Patch Antenna with the Ground Planes}

Typically, for the base station antennas, it is desirable to have dual-polarization [17]. In order to validate that our concept of back radiation suppression works equally well with the dualpolarization antennas, we have performed additional simulations with exactly the same antenna design but with dual polarization. For operation with two orthogonal linear polarizations, the single-polarized patch antenna element (Fig. 3 ) was modified and a second coaxial feed was added at an angle of $90^{\circ}$ to the first coaxial feed (Fig. 10). This dualpolarized patch antenna was simulated with the same semitransparent and metallic ground planes as was done for the single polarized case. The dimensions of the design were also the same.

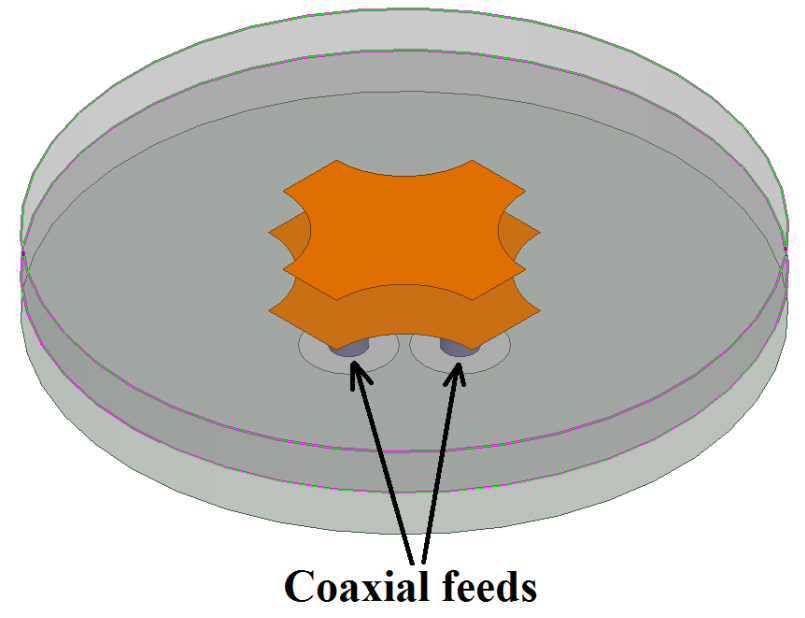

Fig. 10. Dual-polarized stacked patch antenna.

Fig. 11 plots the $S_{11}$ and the isolation $\left(S_{12}\right)$ between the coaxial feeds for the dual-polarized patch antenna. Fig. 11 shows that the bandwidth of the dual-polarized patch antenna is the same as the bandwidth of the single-polarized patch. Also, $\mathrm{S}_{12}$ is less than $-17.6 \mathrm{~dB}$ inside the band $27-35.4 \mathrm{GHz}$ and does not get affected by changing the ground plane from metallic to semitransparent.

Figs. 12 and 13 plot the simulated radiation patterns of the dual-polarized patch antenna with the ground planes at frequencies of 30 and $27 \mathrm{GHz}$. Note, that radiation patterns in the E- and H-planes are the same for each of the linear polarizations. The simulation results show that the radiation patterns of the dual-polarized design at frequencies of 27 and $30 \mathrm{GHz}$ have the same shapes as in Fig. 5-9. Consequently, all conclusions regarding FBR and back radiation suppression for 
the dual-polarized design are the same as that for the singlepolarized design.

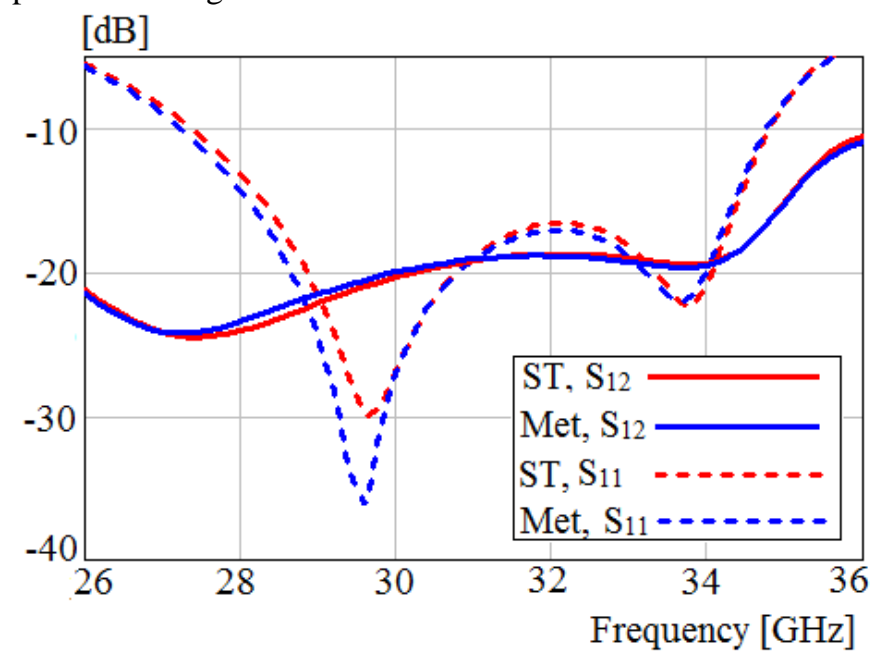

Fig. 11. $S_{11}$ and $S_{12}$ of the dual-polarized patch antenna with the ground planes.

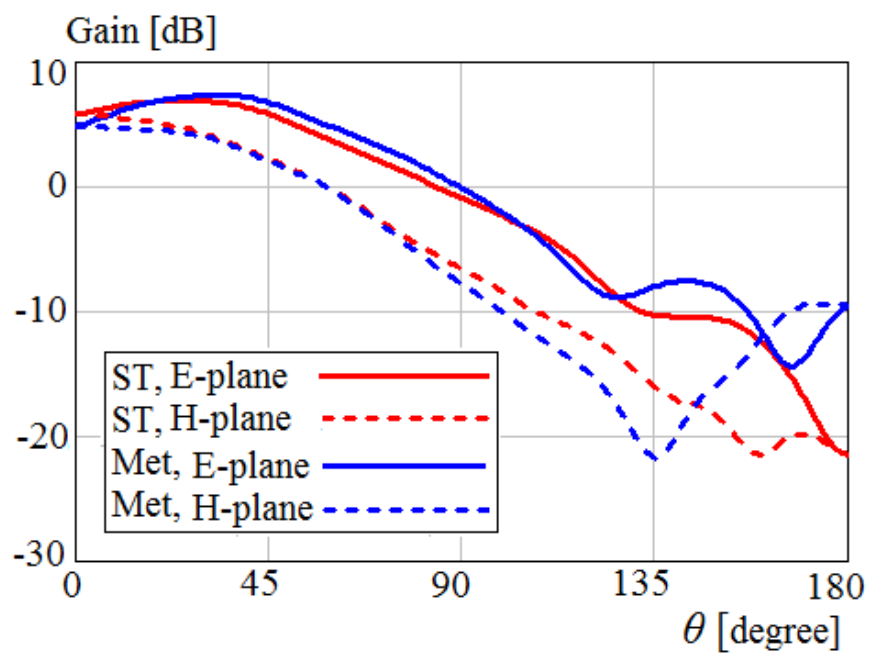

Fig. 12. Radiation patterns of the dual-polarized patch antenna with the ground planes at $30 \mathrm{GHz}$.

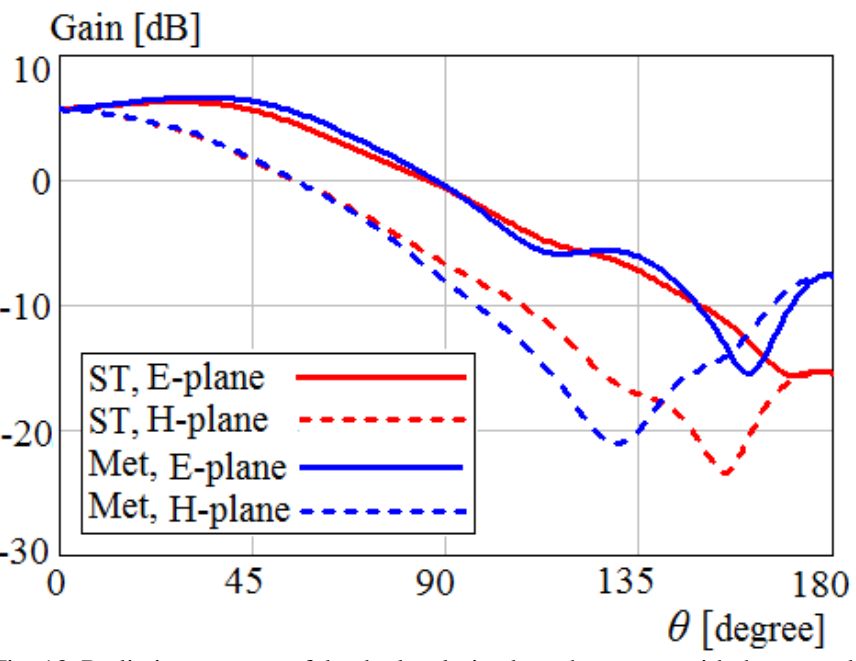

Fig. 13. Radiation patterns of the dual-polarized patch antenna with the ground planes at $27 \mathrm{GHz}$.

\section{CONCLUSIONS}

Using the model of the scattering of a toroidal wave by a round, semitransparent ground plane for the optimization of transparency of the ground plane has allowed us to determine the value of uniform isotropic resistive impedance that provides low level backward radiation of the patch antenna with the ground plane. The increase of the FBR is 11.6 and $8.9 \mathrm{~dB}$ for the patch antenna with the semitransparent ground plane of radius $0.8 \lambda$ and $0.72 \lambda$ respectively, in comparison with the metallic ground plane of the same radius. The proposed patch antenna with the semitransparent ground plane can operate with two orthogonal linear polarizations and can be used as a base station antenna.

\section{ACKNOWLEDGEMENT}

This publication is based upon work supported by the King Abdullah University of Science and Technology (KAUST) Office of Sponsored Research (OSR) under Award No. OSR2016-KKI-2899.

\section{REFERENCES}

[1] Keith Q. T. Zhang, Wireless Communications: Principles, Theory and Methodology. New Jersey: John Wiley and Sons, 2016.

[2] Fa-Long Luo and Charlie Zhang, Signal Processing for 5G: Algorithms and Implementations. New Jersey: John Wiley and Sons, 2016.

[3] Fei Hu, Opportunities in 5G Networks: A Research and Development Perspective. Boca Raton, FL: CRC Press, 2016.

[4] Yi Han, Yan Chen, Beibei Wang, and K. J. Ray Liu, "Time-Reversal Massive Multipath Effect: A Single-Antenna "Massive MIMO" Solution," IEEE Trans. Communications., vol. 64, no. 8, pp. 3382-3394, Aug. 2016.

[5] V. Kaloshin and K. Klionovski, "Scattering of the Field of a Ring Current by a Perfectly Conducting Disk," Journal of Communications Technology and Electronics, vol. 59, no. 6, pp. 481-495, June 2014.

[6] V. Kaloshin and K. Klionovski, "On Radiation of Omnidirectional Axisymmetric Antennas with Circular Ground Planes," Journal of Communications Technology and Electronics, vol. 60, no. 10, pp. 10621071, Oct. 2015.

[7] B. G. Westfall, "Antenna with R-card Ground Plane," U.S. Patent 5694136, Dec. 02, 1997.

[8] B. Rama Rao and E. N. Rosario, "Electro-textile ground planes for multipath and interference mitigation in GNSS antennas covering 1.1 to 1.6 GHz," in Proc. 24th Int. Tech. Meeting Satellite Div. Inst. of Nav. (ION GNSS '11), Portland, OR, USA, 2011, pp. 732-745.

[9] K. Klionovski, "Theoretical and Experimental Research of Diffraction on Round Semitransparent Ground Plane," IEEE Trans. Antennas Propag., vol. 61, no. 6, pp. 3207-3215, June 2013.

[10] Li Du and Yunqi Fu, "A Small Wideband Low-Multipath GNSS Antenna Using Resistive Film," Antennas Wireless Propag. Lett., vol. 12, pp. 1045-1048, 2013.

[11] B. Rama Rao and E. N. Rosario, "Conformal Electro-Textile Antenna and Electronic Band Gap Ground Plane for Suppression of Back Radiation From GPS Antennas Mounted on Aircraft," U.S. Patent application 2014/0022139 A1, Jan. 23, 2014.

[12] R. Garg, P. Bhartia, I. Bahl, and A. Ittipihoon, Microstrip Antenna Design Handbook. Norwood, MA: Artech House, 2001.

[13] V. Kaloshin and K. Klionovski, "Radiation of a Ring Current above a Semitransparent Disk," in Proc. Asia-Pacific Microwave Conf. (APMC 2014), Sendai, Japan, 2014, pp. 1414-1416.

[14] 5G Americas. (2015, August). 5G Spectrum Recommendations. White paper. [Online]. Available: http://www.5gamericas.org/files/6514/3930/9262/4G_Americas_5G_Sp ectrum_Recommendations_White_Paper.pdf 
[15] V. V. Golovin and Y. N. Tyschuk, "Multielement patch antenna array of operated polarization of Ku-band," in Proc. 9th Int. Conf. on Antenna Theory and Techniques (ICATT), Odessa, Ukraine, 2013, pp. 286-288.

[16] M. W. Hyde IV, M. J. Havrilla, and P. E. Crittenden, "A Novel Method for Determining the R-Card Sheet Impedance Using the Transmission Coefficient Measured in Free-Space or Waveguide Systems," IEEE Trans. Instrum. Meas., vol. 58, no. 7, pp. 2228-2233, July 2009.

[17] I. Oshima, "Development of base station antennas for $5 \mathrm{G}$ mobile communication systems," in Proc. IEEE iWEM, Nanjing, China, 2016.

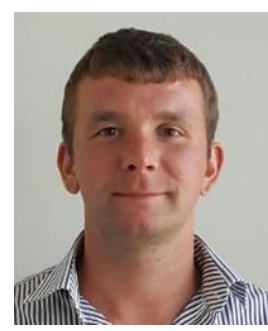

Kirill Klionovski received the Engineer degree (with honor) in electrical engineering from the Moscow Aviation Institute, Moscow, Russia, in 2010 and the Candidate of Sciences degree (is equivalent to $\mathrm{Ph} . \mathrm{D}$.) in radiophysics from Kotel'nikov Institute of Radio-engineering and Electronics of Russian Academy of Sciences, Moscow, Russia, in 2015.

His research interests include the theory of diffraction and asymptotic techniques in applied electromagnetics.

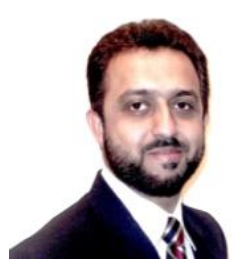

Atif Shamim received his M.A.Sc. and Ph.D degrees in electrical engineering at Carleton University, Canada in 2004 and 2009 respectively.

$\mathrm{He}$ was an NSERC Alexander Graham Bell Graduate scholar at Carleton University from 2007 till 2009 and an NSERC postdoctoral Fellow in 2009-2010 at Royal Military College Canada and KAUST. In August 2010, he joined the Electrical Engineering Program at KAUST, where he is currently an Associate Professor and principal investigator of IMPACT Lab. He was an invited researcher at the VTT Micromodules Research Center (Oulu, Finland) in 2006.

Prof. Shamim was the recipient of the best paper prize at the European Microwave Association Conference in 2008. He was given the Ottawa Centre of Research Innovation (OCRI) Researcher of the Year Award in 2008 in Canada. His work on Wireless Dosimeter won the ITAC SMC Award at Canadian Microelectronics Corporation TEXPO in 2007. Prof. Shamim's students won the Best Paper Prize (3rd position) in IEEE IMS 2016 and (1st position) in IEEE MECAP 2016. Prof. Shamim also won numerous entrepreneurial awards, including 1st prize in Canada's national business plan competition and was selected for OCRI Entrepreneur of the year award in 2010. He is an author/co-author of over 150 international publications and an inventor on 17 US and worldwide patents. His research interests are in integrated antennas and circuits designs for flexible and wearable wireless sensor systems through additive manufacturing techniques such as inkjet printing. Prof. Shamim is a Senior Member of IEEE and serves on the editorial board of IEEE Transactions on Antennas and Propagation. 